\title{
Verified Solution to Optimal Control Problems of Elastic Rod Motion Based on the Ritz Method*
}

\author{
Georgy Kostin ${ }^{a}$
}

\begin{abstract}
To model vibrations in flexible structures, a variational formulation of PDE control problems is considered in the frame of the method of integrodifferential relations. This approach allows to estimate a posteriori the quality of finite-dimensional approximations and, as a result, either to refine or coarsen them if necessary. Such estimates also make it possible to correct the input signals. The related control law is regularized via a quadratic cost functional including the discrepancy of the constitutive equations. Procedures for solving optimization problems in dynamics of linear elasticity have been developed based on the Ritz method and FEM. The verification of optimized control for elastic rod motion involves the local and integral error estimates proposed. A FEM solver for mechanical systems with varying distributed parameters and linear boundary conditions of different kinds is presented.
\end{abstract}

Keywords: optimal control, dynamics, systems with distributed parameters, elasticity, Ritz method, finite element method

\section{Introduction}

An actual direction of control theory is optimization of motion for systems with distributed parameters. The mathematical basis for control problems described by linear partial differential equations (PDEs) and convex functionals was laid by Lions [21]. Hyperbolic equations are treated in [1, 6]. An introduction to the control of vibrations can be found in [17]. Oscillating elastic networks are investigated in $[12,18,19]$. Reliable modelling of such systems leads to specific approaches for solving direct and inverse problems. Two alternate methods in control design for spatially distributed processes can be mentioned. In the first one, so-called late lumping, the optimal control law is directly derived for the original PDE models and afterwards approximated by finite-dimensional functions. The functional analysis can rely on spectrum theory of linear operators $[4,9]$. The

*The study has been done under financial support of the Russian Science Foundation (grant 16-11-10343).

${ }^{a}$ Ishlinsky Institute for Problems in Mechanics of the Russian Academy of Sciences, E-mail: kostin@ipmnet.ru 
control decomposition proposed in [7] on the basis of the Fourier approach allows to construct a constrained control that brings the dynamic system to a given state in limited time. An approach to PDE-constrained optimal control problems is adapted in [11] for hyperbolic equations and related with the method of choice.

The second method with early lumping is often used for numerical control design. According to this methodology, the initial-boundary value problem is first discretized and reduced to a system of ordinary differential equations (ODEs). As pointed out in [20], the finite element method (FEM) as well as finite volume and finite difference schemes $[3,8]$ can be adapted for this purpose. If FEM are applied, collocation methods, modifications of the Galerkin method [5], its PetrovGalerkin versions [2], or least-squares techniques [13] are often used in applications. If eigenfunctions are available, a Fourier analysis can also be very efficient [9]. To model vibrations in flexible structures, variational formulations of control problems with PDEs were proposed by using the method of integro-differential relations (MIDR) [16]. By introducing integral constitutive relations, this approach gives the possibility to estimate the quality of a finite-dimensional model, to refine or coarsen the obtained approximations if necessary, and to correct the related control law. An effective algorithm for solving optimal control problems in linear elasticity based on the Ritz method and the FEM was proposed in $[14,15]$, for uniform elastic rods. As shown, the accuracy of the approximate solution can dramatically fall down after control optimization. To regulate the appearing error, the constitutive functional is constrained by an isoperimetric error condition.

The novelty of the presented study can be summarized with the following sentences. As compared with $[14,15]$, the variational formulation of initial-boundary value problems in linear elasticity, which is based on the integral representation of constitutive relations proposed by authors, is extended to the case of nonuniform structures including the elements of different dimensions. Affine boundary conditions of various kinds are considered in the new variational statement either as natural or as essential constraints. The originality of this paper is also in developing FEM procedures specially for these boundary conditions with piecewise polynomial approximations in the space-time domain for unknown displacement, momentum, and force fields. The optimization of elastic rod motions is performed by taking into account variable mechanical parameters and boundary conditions of the third kind (elastic support). The control law depressing undesired vibrations in the rod is found with minimization of both mean and terminal energies over a fixed time horizon. The strategy of feedforward control is generalized to the case of multiple input system with different scheme of control parametrization depending on the classes of excitations (kinematic or dynamic). It has been found the exact optimal motion of the uniform elastic rod controlled at both ends and proved the invariance of this solution with respect to the type of boundary conditions and weighting coefficients in the objective functional. The applicability of the numerical algorithm is demonstrated on an example of a rod with piecewise constant parameters.

The paper is orginized as follows. In Sect. 2, an optimal control problems is formulated. The FEM algorithm is described in Sect. 3. The numerical results is discussed in Sect. 4. A short conclusions and outlook is given in Sect. 5. 


\section{Controlled dynamics of elastic structures}

\subsection{Longitudinal motions of a thin elastic rod}

Let us consider an initial-boundary value problem of longitudinal motions for a thin rectilinear elastic rod (see Fig. 1). The PDEs describing motions of the dynamic system with distributed parameters can be split into two parts. The first group is the constitutive relations

$$
p(t, x)=\rho(x) w_{t}(t, x) \quad \text { and } \quad s(t, x)=\kappa(x) w_{x}(t, x)
$$

between the momentum density $p(t, x)$ and the velocities as well as between the normal forces $s(t, x)$ at the cross section and the material strains. Here, $\kappa(x)$ is the tension stiffness, and $\rho(x)$ is the linear density. The function $w(t, x)$ defined on the time-space domain $\Omega=(0, T) \times\left(x^{0}, x^{1}\right)$ is the displacements of the rod points.

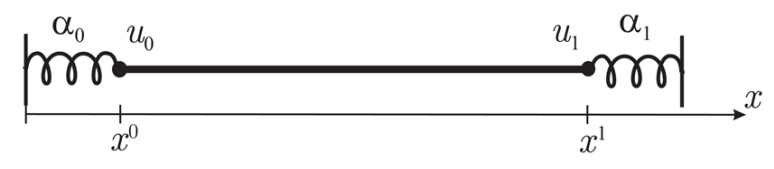

Figure 1: Scheme of a rectilinear rod with elastic supports.

One PDE joints the momenta and forces according to Newton's second law

$$
p_{t}(t, x)=s_{x}(t, x)+f(t, x),
$$

where $f(t, x)$ is the linear density of external loads. The initial conditions in this statement are imposed on both the displacements and the momentum density by

$$
w(0, x)=w_{0}(x) \text { and } p(0, x)=p_{0}(x) .
$$

Finally, the nonhomogeneous (affine) boundary conditions of the third kind are defined as follows

$$
\cos \alpha_{i} s\left(t, x^{i}\right)=(-1)^{i} \chi \sin \alpha_{i} w\left(t, x^{i}\right)+u_{i}(t) \text { for } i=0,1 \text { with } 0 \leq 2 \alpha_{i} \leq \pi .
$$

Here, two values $\alpha_{i}$ are introduced so that the coefficient $\chi_{i}=\chi \tan \alpha_{i}$ means the stiffness of a lumped spring placed at $x=x^{i}$ with the characterictic stiffness of the rod $\chi=\left(x^{1}-x^{0}\right)^{-2} \int_{x^{0}}^{x^{1}} \kappa(x) \mathrm{d} x$. The control value $u_{i}(t)$ defines either the displacement $w$ if $2 \alpha_{i}=\pi$ or the force $s$ if $\alpha_{i}=0$. In all the other cases, relations (4) represent elastic supports at the rod ends [10]. This expression is not unique but helps to describe regularly a rather wide class of linear boundary conditions.

\subsection{Variational principle in structural dynamics}

To state the variational principle in dynamics for elastic structures in accordance with the MIDR [16], an integral representation of the constitutive laws is used 
instead of the differential relations (1). For this purpose, scaled functions of the constitutive laws

$$
g:=\sqrt{\rho} w_{t}-\sqrt{\rho^{-1}} p \quad \text { and } \quad h:=\sqrt{\kappa} w_{x}-\sqrt{\kappa^{-1}} s
$$

are first introduced. This leads to the modified constitutive relations

$$
g(t, x)=0 \quad \text { and } \quad h(t, x)=0 .
$$

Two auxiliary functions of relative displacements $q(t, x)$ and dynamic potential $r(t, x)$ are then defined such that the change of the unknown variables

$$
\begin{aligned}
& w(t, x)=q(t, x)+w_{0}(x), \quad s(t, x)=r_{t}(t, x), \\
& p(t, x)=r_{x}(t, x)+p_{0}(x)+\int_{0}^{t} f(\tau, x) \mathrm{d} \tau
\end{aligned}
$$

is performed. This transformation satisfies automatically the balance equation (2).

The variational reformulation of the direct dynamic problem (1)-(4) with respect to the new variables, kinematic $q(t, x)$ and dynamic $r(t, x)$, can be given as follows. Find such functions $q^{*}(t, x)$ and $r^{*}(t, x)$ in the Hilbert space $H_{1}(\Omega)$ which minimize the constitutive functional

$$
\begin{gathered}
J_{0}\left[q^{*}, r^{*}, u_{0}, u_{1}\right]=\min _{q, r \in H^{1}(\Omega)} J_{0}\left[q, r, u_{0}, u_{1}\right]=0, \\
J_{0}=\frac{1}{T} \int_{\Omega} \varphi(t, x) \mathrm{d} \Omega+\frac{1}{2 \chi T} \sum_{k=0}^{1} \int_{0}^{T} \beta_{k} h_{k}^{2}(t) \mathrm{d} t, \quad \varphi:=\frac{g^{2}}{2}+\frac{h^{2}}{2}, \\
h_{i}(t)=\cos \alpha_{i} r_{t}\left(t, x^{i}\right)-(-1)^{i} \chi \sin \alpha_{i}\left(q\left(t, x^{i}\right)+w_{0}\left(x^{i}\right)\right)-u_{i}(t), \quad i=0,1 .
\end{gathered}
$$

In the numerical realization discussed below, the nonzero value of $J_{0}$ estimates the integral error of approximate solutions, whereas the integrand $\varphi$ can be utilized for local error estimates. The indicators $\beta_{i}:=\operatorname{sgn} \sin 2 \alpha_{i}$ define whether the boundary relations are considered as essential constraints (rigid or free support, $\beta_{i}=0$ ) or as generalized natural conditions (proper elastic support, $\beta_{i}=1$ ).

The minimization is subject to the initial and boundary constraints with respect to both the kinematic variable $q$ and the dynamic one $r$ in the form

$$
q(0, x)=0 \quad \text { and } \quad r(0, x)=0 ; \quad\left(1-\beta_{i}\right) h_{i}(t)=0 \text { for } i=0,1 .
$$

\subsection{Optimal control problem}

The following optimal control problem is considered with weighted minimization of the mean and terminal energies of the rod. Find control functions $u_{i}^{*}(t)$ for $i=0,1$ such that the energy functional $J$ reaches its minimum over a fixed time horizon as

$$
\begin{gathered}
J\left[w, p, s, u_{0}^{*}, u_{1}^{*}\right]=\min _{u_{0}, u_{1} \in L_{2}(0, T)} J\left[w, p, s, u_{0}, u_{1}\right], \quad J=\gamma_{1} J_{1}+\gamma_{2} J_{2}, \\
J_{1}=\frac{1}{T} \int_{0}^{T} W(t) \mathrm{d} t, \quad J_{2}=W(T), \quad \bar{\chi}_{i}=\left\{\begin{array}{ll}
\chi_{i}, & 2 \alpha_{i} \neq \pi \\
0, & 2 \alpha_{i}=\pi
\end{array},\right. \\
W(t)=\frac{1}{2} \int_{x^{0}}^{x^{1}}\left(\kappa w_{x}^{2}+\rho^{-1} p^{2}\right) \mathrm{d} x+W_{0}(t)+W_{1}(t), \quad W_{i}(t)=\frac{\bar{\chi}_{i} w^{2}\left(t, x^{i}\right)}{2}
\end{gathered}
$$


subject to the PDE constraints (1)-(4). Here, $W(t)$ is the potential and kinetic energy of the rod and the end springs if any, $J_{1}$ denotes the mean energy of the system during the process, $J_{2}$ is the whole energy at the terminal time instant $T$. It is worth noting that the weight coefficient $\gamma_{1}$ is assumed to be positive to avoid ill-definedness of the control problem, whereas $\gamma_{2} \geq 0$ can be equated to zero.

The problem is directly generalized in the frame of the variational formulation given in the previous subsection. For this purpose, the change of variables (7) in the functional $J$ is first performed and the differential constraints (1)-(4) are then replaced for relations (8), (9).

\section{$3 \quad$ FEM based on the Ritz method}

\subsection{Piecewise polynomial approximations}

Regular triangulation of the space-time domain $\Omega$ in the frame of a finite-element algorithm is described in detail in [16]. Here, just the main idea of discretization is given without going into particulars. In the presented study, only meshes with the uniform partition in time and space are utilized. Piecewise polynomial splines are involved for approximation of unknown functions

$$
\tilde{q}=\sum_{k+l \leq K} q_{m n j}^{(k l)} t^{k} x^{l} \quad \text { and } \quad \tilde{r}=\sum_{k+l \leq K} r_{m n j}^{(k l)} t^{k} x^{l} \quad \text { for } \quad(t, x) \in \Delta_{m n j} .
$$

Here, $K$ denotes the polynomial degree and $\{k, l\} \subset \mathbb{N}_{0}$. The triangular subdomains $\Delta_{m n j}$ of the mesh with the indices $j=1, \ldots, 4, m=1, \ldots, M, n=1, \ldots, N$ are shown in Fig. 2.

The mesh is defined by the nodes on the $t$-axis and $x$-axis respectively as

$$
\begin{cases}x_{m}=x^{0}+M^{-1} m\left(x^{1}-x^{0}\right) & \text { for } \quad m=0, \ldots, M \\ t_{n}=N^{-1} n T & \text { for } \quad n=0, \ldots, N\end{cases}
$$

where $M$ and $N$ are the only mesh parameters. The domain $\Omega$ is subdivided by the straight lines $x=x_{m}$ and $t=t_{n}$ into $M N$ rectangles $\Omega_{m n}=\left(t_{n-1}, t_{n}\right) \times\left(x_{m-1}, x_{m}\right)$ with $m=1, \ldots, M$ and $n=1, \ldots, N$. The diagonals of $\Omega_{m n}$ cut this rectangle into four triangles $\Delta_{m n j}, j=1, \ldots, 4$. The total number of parameters $q_{m n j}^{(k l)}$ and $r_{m n j}^{(k l)}$ for each mesh element $\Delta_{m n j}$ is equal to $2 N_{\Delta}=(K+1)(K+2)$.

The column vector $\hat{\mathbf{z}} \in \mathbb{R}^{N_{l}}$ is introduced including all the local parameters $q_{j m n}^{(k l)}, r_{j m n}^{(k l)}$ with the dimension $N_{l}=8 M N N_{\Delta}$. The tuples $\hat{\mathbf{q}}$ and $\hat{\mathbf{r}}$ of discontinuous basis functions are defined on $\Omega$ so that $\hat{q}=\hat{\mathbf{q}}^{\mathrm{T}} \hat{\mathbf{z}}$ and $\hat{r}=\hat{\mathbf{r}}^{\mathrm{T}} \hat{\mathbf{z}}$ correspond to the unknown functions $\tilde{q} \in C(\Omega)$ and $\tilde{r} \in C(\Omega)$. Thus, they have to satisfy the initial and boundary constraints (9) as well as the interface continuous conditions in accordance with (8).

After the essential conditions are all imposed on $q(t, x)$ and $r(t, x)$, we can construct the matrix $\mathbf{Q} \in \mathbb{R}^{N_{l} \times N_{g}}$ with $N_{g}=4 M N K^{2}+\left(\beta_{0}+\beta_{1}\right) K N$ which ties 


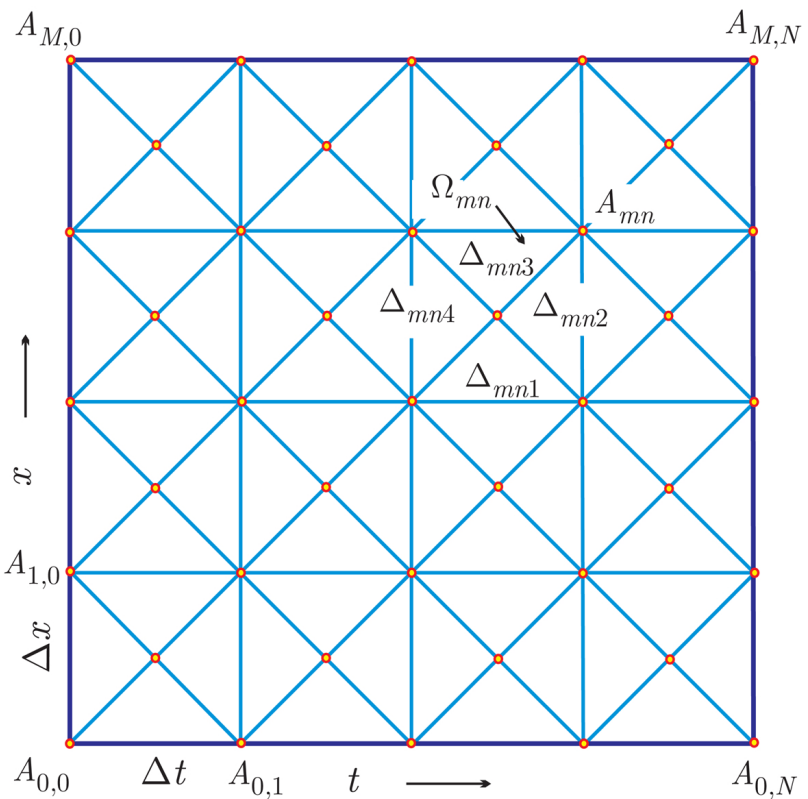

Figure 2: Regular triangulation of the space-time domain.

the original vector $\hat{\mathbf{z}}$ with the resulting independent parameters $\mathbf{z}$ by $\hat{\mathbf{z}}=\mathbf{Q z}$. The unknowns are then represented in the form

$$
\tilde{q}(t, x)=\mathbf{z}^{\mathrm{T}} \mathbf{q}(t, x) \quad \text { and } \quad \tilde{r}(t, x)=\mathbf{z}^{\mathrm{T}} \mathbf{r}(t, x) \quad \text { with } \quad \mathbf{z} \in \mathbb{R}^{N_{g}} .
$$

\subsection{Regularization of the optimal control problem}

As shown in [15], the error of the approximation (12) can increase after control optimization. To limit the error range and to enhance thus the reliability of modelling, the value of the constitutive functional $J_{0}$ can be fixed by the isoperimetric condition

$$
\bar{J}_{0}\left[\mathbf{z}, u_{0}, u_{1}\right]=\varepsilon \ll \bar{J}_{2}\left[\mathbf{z}, u_{0}, u_{1}\right] \ll \bar{J}_{1}\left[\mathbf{z}, u_{0}, u_{1}\right],
$$

where $\bar{J}_{i}\left[\mathbf{z}, u_{0}, u_{1}\right]:=J_{i}\left[\tilde{q}, \tilde{r}, u_{0}, u_{1}\right]$ for $i=0,1,2$ with the functions $\tilde{q}, \tilde{r}$ from (12).

In accordance with the Lagrange theorem [22], the isoperimetric condition (13) leads us to the corresponding unconstrained control problem: Find the functions $u_{0}^{*}(t)$ and $u_{1}^{*}(t)$ that minimize the modified cost functional

$$
\begin{gathered}
\bar{J}\left[\mathbf{z}^{*}, u_{0}^{*}, u_{1}^{*}\right]=\min _{u_{0}, u_{1} \in L_{2}(0, T)} \bar{J}\left[\overline{\mathbf{z}}\left[u_{0}, u_{1}\right], u_{0}, u_{1}\right], \\
\bar{J}=\gamma_{0} \bar{J}_{0}+\gamma_{1} \bar{J}_{1}+\gamma_{2} \bar{J}_{2}, \quad \gamma_{1}>0, \quad \gamma_{l} \geq 0, l=0,2, \quad \mathbf{z}^{*}:=\overline{\mathbf{z}}\left[u_{0}^{*}, u_{1}^{*}\right] .
\end{gathered}
$$

For arbitrary control signals $u_{0}, u_{1}$, the vector

$$
\overline{\mathbf{z}}\left[u_{0}, u_{1}\right]=\arg \min _{\mathbf{z}} \bar{J}_{0}\left[\mathbf{z}, u_{0}, u_{1}\right] \geq 0
$$


is the result of optimization of the constitutive functional $\tilde{J}_{0}$. The approximation error can be estimated by the ratio

$$
\Delta=\bar{J}_{0}\left[\mathbf{z}^{*}, u_{0}^{*}, u_{1}^{*}\right] \bar{J}_{1}^{-1}\left[\mathbf{z}^{*}, u_{0}^{*}, u_{1}^{*}\right] .
$$

\subsection{Numerical algorithm of control optimization}

In the presented realization, two finite-dimensional control signals $u_{0}(t)$ and $u_{1}(t)$ are taken as piecewise polynomial functions. The corresponding time mesh has the same nodes $t_{n}$ as in the domain triangulation described in Subsec. 3.1. If $2 \alpha_{i}=\pi$ for $i=0$ or $i=1$ in (4), the boundary displacement $w\left(t, x^{i}\right)$ is defined by a continuous spline with the polynomial order $K$. The control values $u_{i}(t)$ can be represented then on each interval $t \in\left[t_{n-1}, t_{n}\right]$ with the length $\tau=t_{n}-t_{n-1}=T / N$ by the Bernstein polynomials as

$$
\tilde{u}_{i}(t)=\frac{1}{\tau^{K}} \sum_{k=0}^{K} v_{j(i, k, n)} C_{k}^{K}\left(t-t_{n-1}\right)^{k}\left(t_{n}-t\right)^{K-k},
$$

where $n=1, \ldots, N$, and $C_{k}^{K}$ is a binomial coefficient. For all the time interval, the control parameters $v_{j}$ have the through indexation $j(i, k, n)=(i N+n-1) K+$ $k$. The coefficient $v_{i K N}$ is defined unambiguously in accordance with the initial conditions (4), namely, $u_{i}(0)=w^{0}\left(x^{i}\right)$.

When $2 \alpha_{i}<\pi$ for $i=0$ or $i=1$, the control value $u_{i}(t)$ is given, in contrast to (16), by the discontinuous spline of order $K-1$ in the form

$$
\tilde{u}_{i}(t)=\frac{1}{\tau^{K-1}} \sum_{k=0}^{K-1} v_{j(i, n, k)} C_{k}^{K-1}\left(t-t_{n-1}\right)^{k}\left(t_{n}-t\right)^{K-k-1}
$$

for the same time interval and index function $j(i, k, n)$ as for $2 \alpha_{i}=\pi$. Note that each coefficient $v_{j}$ in (17) relates only with one time interval, whereas polynomials in (16) defined on two adjacent intervals $\left[t_{n-1}, t_{n}\right]$ and $\left[t_{n}, t_{n+1}\right]$ have the common coefficient $v_{j(i, K, n)}$ due to the continuity of the displacement field in time. So, let

$$
\mathbf{u}=\left[v_{1} \ldots v_{N_{u}}\right]^{\mathrm{T}} \in \mathbb{R}^{N_{u}}
$$

denote the column vector of control parameters with the dimension $N_{u}=2 K N$.

After substituting either (16) or (17) in the functional $\bar{J}$ from (13), the constitutive function $\tilde{J}_{0}(\mathbf{z}, \mathbf{u}):=\bar{J}_{0}\left[\mathbf{z}, \tilde{u}_{0}, \tilde{u}_{1}\right]$ can be expressed through the vectors of state parameters $\mathbf{z}$ and control parameters $\mathbf{u}$ as follows

$$
\tilde{J}_{0}(\mathbf{y})=\frac{1}{2} \mathbf{y}^{\mathrm{T}} \mathbf{F y}+\mathbf{f}^{\mathrm{T}} \mathbf{y}+f \rightarrow \min _{\mathbf{z}},
$$

where

$$
\mathbf{F}=\left[\begin{array}{ll}
\mathbf{F}_{z z} & \mathbf{F}_{z u} \\
\mathbf{F}_{z u}^{\mathrm{T}} & \mathbf{F}_{u u}
\end{array}\right], \mathbf{f}=\left[\begin{array}{c}
\mathbf{f}_{z} \\
\mathbf{f}_{u}
\end{array}\right], \mathbf{y}=\left[\begin{array}{l}
\mathbf{z} \\
\mathbf{u}
\end{array}\right] .
$$

The minimization of $\tilde{J}_{0}$ with respect to $\mathbf{z}$ gives the best state vector

$$
\tilde{\mathbf{z}}(\mathbf{u})=-\mathbf{F}_{z z}^{-1}\left(\mathbf{F}_{z u} \mathbf{u}+\mathbf{f}_{z}\right) .
$$


By using (21), the cost function $\tilde{J}(\mathbf{u}):=\bar{J}\left[\tilde{\mathbf{z}}, \tilde{u}_{0}, \tilde{u}_{1}\right]$ takes the following structure

$$
\tilde{J}(\mathbf{u})=\frac{1}{2} \mathbf{u}^{\mathrm{T}} \mathbf{V u}+\mathbf{v}^{\mathrm{T}} \mathbf{u}+v \rightarrow \min _{\mathbf{u}} .
$$

The vector of optimal control parameters is derived directly from (22) as

$$
\mathbf{u}^{*}=-\mathbf{V}^{-1} \mathbf{v} .
$$

The control vector $\mathbf{u}^{*}$ defined by (23) generates the corresponding state vector $\tilde{\mathbf{z}}^{*}=\tilde{\mathbf{z}}\left(\mathbf{u}^{*}\right)$ and gives us the control functions $\tilde{u}_{i}^{*}(t)=\tilde{u}_{i}\left(t, \mathbf{u}^{*}\right)$ with $i=0,1$. The kinematic and dynamic functions follow as

$$
\tilde{q}^{*}(t, x)=\tilde{q}\left(t, x, \tilde{\mathbf{z}}^{*}, \mathbf{u}^{*}\right) \quad \text { and } \quad \tilde{r}^{*}(t, x)=\tilde{r}\left(t, x, \tilde{\mathbf{z}}^{*}, \mathbf{u}^{*}\right) .
$$

Finally, the approximate solution of the original control problems is obtained according to the change of variables (7) by

$$
\tilde{w}^{*}(t, x)=w\left(x, \tilde{q}^{*}(t, x)\right), \quad \tilde{p}^{*}(t, x)=p\left(t, x, \tilde{r}^{*}(t, x)\right), \quad \tilde{s}^{*}(t, x)=s\left(\tilde{r}^{*}(t, x)\right) .
$$

\section{Results of optimization}

\subsection{Exact solution for the uniform rod}

Introducing new coordinates and variables as

$$
\begin{aligned}
& x=x_{0}+L x^{*}, \quad t=\tau_{0} t^{*}, \quad w(t, x)=L w^{*}(t, x), \\
& \rho(x)=\bar{\rho} \rho^{*}(x), \quad \kappa(x)=\bar{\kappa} \kappa^{*}(x), \quad f(t, x)=L^{-1} \bar{\kappa} f^{*}(t, x), \\
& \tau_{0}=L \sqrt{\frac{\bar{\rho}}{\bar{\kappa}}}, \quad \bar{\rho}=\frac{1}{L} \int_{x^{0}}^{x^{1}} \rho(x) \mathrm{d} x, \quad \bar{\kappa}=\frac{1}{L} \int_{x^{0}}^{x^{1}} \kappa(x) \mathrm{d} x,
\end{aligned}
$$

we turn to a dimensionless form of the rod's dynamic equations (1)-(4). Here, $L=x_{1}-x_{0}$ denotes the length of the rod, $\tau_{0}$ is the characteristic time, $\bar{\rho}$ and $\bar{\kappa}$ are the mean linear density and stiffness, respectively. In the case when $\rho=$ const and $\kappa=$ const, $\rho^{*}=\kappa^{*}=1$ after such change of variables. For simplicity, the star superscript will be omitted in what follows.

A uniform elastic rod with the dimensionless parameters $\rho=\kappa=x^{1}=1$ and $x^{0}=0$ is first considered. Thus, the effective stifness of the rod is $\chi=1$. As an example of the systems with the boundary conditions of the third kind in (4), we choose $\alpha_{0}=\pi / 3$ at $x=0$ and $\alpha_{1}=\pi / 6$ at $x=1$. This means that

$$
\sqrt{3} s(t, 0)=w(t, 0)+2 u_{0}(t) \quad \text { and } \quad s(t, 1)=-\sqrt{3} w(t, 1)+2 u_{1}(t) .
$$

We suppose from here that the distributed external force vanishes, in other words, $f(t, x) \equiv 0$. Without loss of generality, the terminal state is fixed to zero:

$$
w(T, x)=p(T, x)=0 \quad \text { for } \quad x \in(0,1) .
$$


It is always possible for the control time horizon $T \geq 1$ to find the exact control displacement $\bar{u}_{0}(t)$ and force $\bar{u}_{1}(t)$ leading the rod from the initial state $w_{0}(x)$, $p_{0}(x)$ to any desired terminal state [6]. If $T=1$, the admissible control law is unique and given by

$$
\begin{aligned}
& 2 \bar{u}_{0}(t)=\sqrt{3} a_{+}^{\prime}(t)-a_{+}(t) \quad \text { and } \quad 2 \bar{u}_{1}(t)=-a_{-}^{\prime}(t-1)+\sqrt{3} a_{-}(t-1) \\
& \text { with } \quad a_{ \pm}(x)=\frac{w_{0}(x) \pm r_{0}(x)}{2} \text { and } r_{0}(x)=-\int_{x}^{1} p_{0}(\xi) \mathrm{d} \xi .
\end{aligned}
$$

It is follows from the expressions for the first and second variations of the cost functional $J$ that the common control function

$$
u_{i}^{*}(t)=\left\{\begin{array}{ll}
\bar{u}_{i}(t) & \text { for } t \in[0,1] \\
0 & \text { for } t>1
\end{array} \quad \text { with } \quad i=0,1\right.
$$

is optimal to the problem (8)-(10) (without terminal constraints) for any weight coefficients $\gamma_{1}>0, \gamma_{2} \geq 0$ and any time instant $T>0$. As an example, the initial trigonometric displacements $w_{0}(x)=\sin (\pi x)$ and the zero momentum density $p_{0}(x) \equiv 0$ are taken. The optimal displacements $w(t, x)$ shown in the dimensionless form in Fig. 3 are invariant of the boundary parameters $\alpha_{i}$ for $i=0,1$, and only the control functions $u_{i}$ change according to (25). Note that the corresponding force field $s(t, x)$ is discontinuous as presented in Fig. 4.

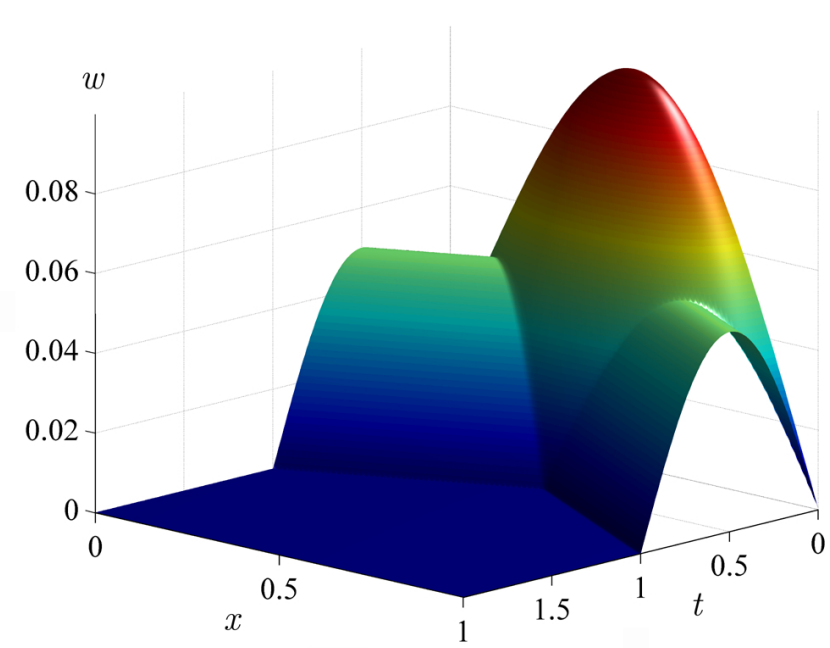

Figure 3: Optimal displacements for the uniform rod. 


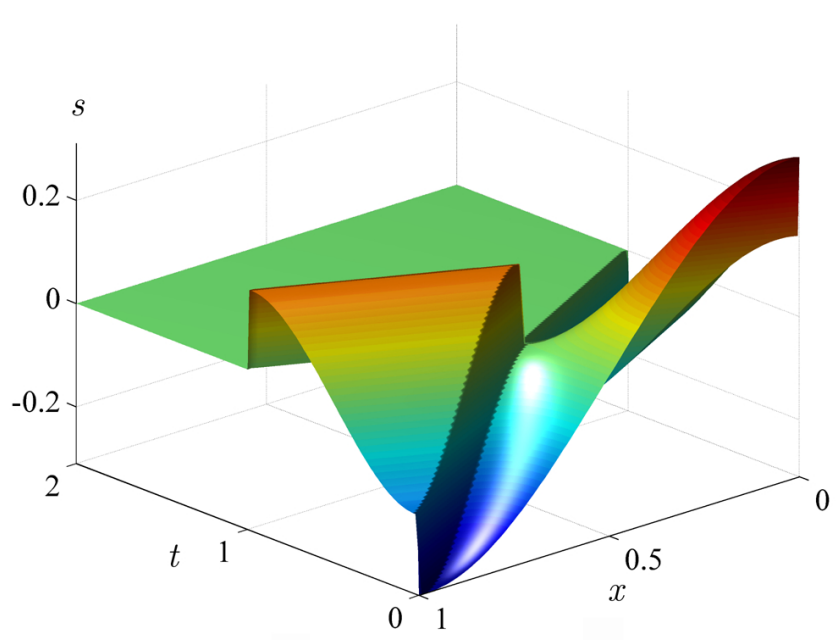

Figure 4: Optimal forces for the uniform rod.

\subsection{Numerical solution for a non-uniform rod}

The case of the nonuniform rod is studied in this subsection for illustration. The piecewise constant density in the dimensionless form is chosen as follows

$$
\rho(x)=\left\{\begin{array}{ll}
\rho_{1}=2.25 & \text { for } x<0.5 \\
\rho_{2}=0.25 & \text { for } x>0.5
\end{array} .\right.
$$

The constant stiffness $\kappa(x)=1$ and the length $L=1$ are taken. The control parameters are fixed as $T=2, \gamma_{1}=1, \gamma_{2}=10$. Similar to the uniform rod discussed in the previous subsection, the mechanical and geometrical constants are chosen so that the time of the signal propagation from one end of the elastic rod to the other is equal to $\tau_{0}=\left(\sqrt{\rho_{1}}+\sqrt{\rho_{2}}\right) / 2=1$. Again, the boundary conditions (24) are taken. The mesh sizes are $N=4$ in time and $M=2$ in space, while the polynomial degree is $K=4$. The resulting number of approximation degrees of freedom is $N_{g}=512$. The dimension of the control vector is $N_{u}=32$. The same as before, the modelling qualities are calculated for the initial displacements $w_{0}(x)=\sin (\pi x)$ and the momentum density $p_{0}(x) \equiv 0$.

On the first stage, the zero weight coefficient $\gamma_{0}=0$ at the energy error term $\tilde{J}_{0}$ is fixed in the cost functional $\tilde{J}$ introduced in (14). For the given parameters, the mechanical energy becomes small enough during the final stage of the optimized motion and reaches its terminal value $\tilde{J}_{2}=5.90 \cdot 10^{-6}$. At that, the mean energy of the system equals to $\tilde{J}_{1}=0.0082$. One of the control signals, namely, $\tilde{u}_{0}^{*}(t)$ at $x=0$ is shown in Fig. 5 by the dashed curve. In turn, the second signal $\tilde{u}_{1}^{*}(t)$ at $x=1$ is depicted in Fig. 6 (also by the dashed curve). 


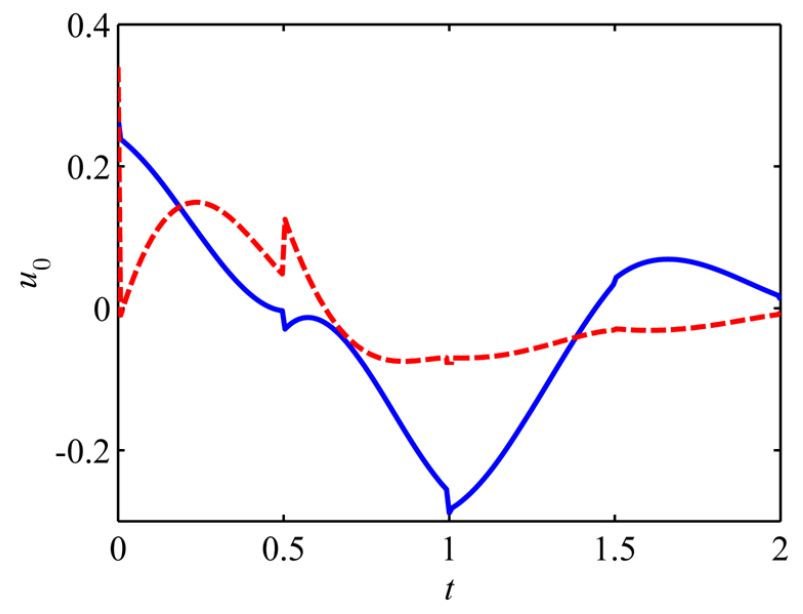

Figure 5: Control signal $\tilde{u}_{0}^{*}(t)$ for $\gamma_{0}=0$ (dashed) vs. $\gamma_{0}=10^{6}$ (solid).

The displacement $\tilde{w}(T, x)$ at the terminal instant is close to zero. However, what has been obtained is a poorly approximate solution. Indeed, the constitutive functional is equal to $\tilde{J}_{0}=1.027 \cdot 10^{-4}$ for $\gamma_{0}=0$. The relative error is $\Delta=0.63 \%$ for the chosen mesh and the fixed polynomial degree. The distribution of the error function $\varphi(t, x)$ is presented in Fig. 7. The error reaches its maximum at $x=1$ in the beginning of the control process. The magnitude of $\tilde{J}_{0}$ does not fulfil the inequality (13) (quite the contrary, $\tilde{J}_{0} \gg \tilde{J}_{2}$ ). Therefore, we cannot consider this approximation as reliable. The accuracy of the terminal energy value $\tilde{J}_{2}$ has to be estimated by taking into account the relatively high level of discretization error.

There are two ways to refine the solution: either by increasing the dimension of approximation or by regularizing the control law, as proposed in Subsec. 3.2. Let us take the weight coefficient at the energy error $\tilde{J}_{0}$ in the cost function $\tilde{J}$ as $\gamma_{0}=10^{4}$. The resulting finite-dimensional functions $\tilde{u}_{0}^{*}(t)$ and $\tilde{u}_{1}^{*}(t)$ are shown by solid curves in Fig. 6 and 7, respectively. The mean value of the system energy, at that, has increased up to $\tilde{J}_{1}=0.0262$. The terminal energy remain small enough $\tilde{J}_{2}=1.78 \cdot 10^{-5} \ll \tilde{J}_{1}$. But the value of the constitutive functional for $\gamma_{0}=10^{4}$ has fallen to $\tilde{J}_{0}=2.23 \cdot 10^{-6}$. The relative error is now $\Delta=4.26 \cdot 10^{-3 \%}$. What seems more important is that the ratios (13) among three functionals are certainly fulfilled.

The displacements $\tilde{w}^{*}(t, x)$ presented in Fig. 8 is continuous by construction. The displacement field has a global maximum at the beginning of the process and some local extrema during the rod motion. The corresponding force field $\tilde{s}^{*}(t, x)$ is shown in Fig. 9.

It is worth emphasizing that the force field $\tilde{s}^{*}(t, x)$ as well as the control function $\tilde{u}_{i}^{*}(t)$ for $i=0,1$ are discontinuous with possible jumps at mesh edges. The control signals are switched at the instant $t \in\{0.5,1,1.5, \ldots\}$. However, such instantaneous 


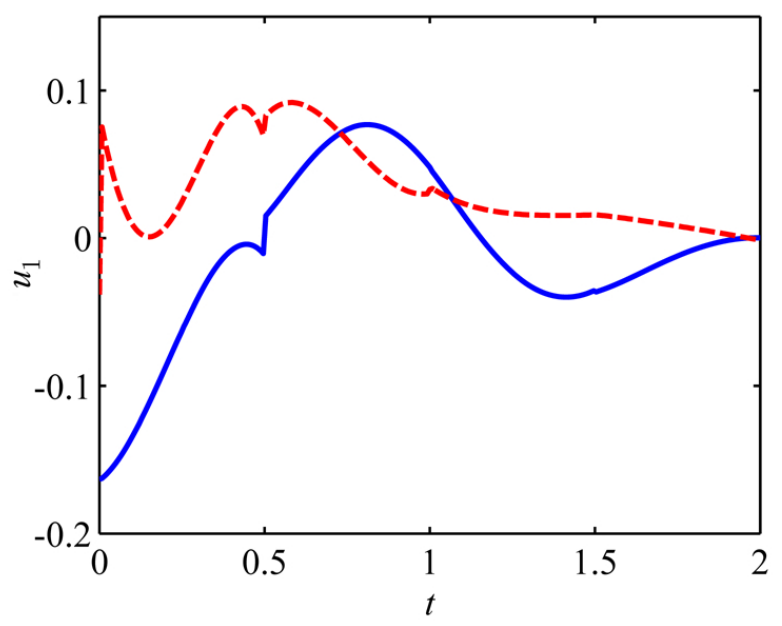

Figure 6: Control signal $\tilde{u}_{1}^{*}(t)$ for $\gamma_{0}=0$ (dashed) vs. $\gamma_{0}=10^{6}$ (solid).

changes after regularization of the optimized control, although presenting, are not significant in magnitude, as seen in Figs. 6 and 7.

As demonstrated by numerical simulation, increasing the dimension of spline approximation and the control dimension does not lead to sufficient minimization of the mean energy for this control process. Further rise of the weight $\gamma_{0}$ leads to numerical instability of the algorithm. A good-tempered choice for the coefficients $\gamma_{i}, i=0,1,2$, remains an object of future study.

\section{Concluding remarks and outlook}

A strategy of energy optimization in structural dynamics has been proposed. As an illustrative example, longitudinal motions of nonuniform elastic rods with kinematic and dynamic control inputs are considered. The optimization algorithm is based on the method of integro-differential relations, which makes it possible to combine directly a variational statement of initial-boundary problems with the finite element method. The verification of optimal control laws is performed by taking into account a posteriori local and integral estimates.

In the future, the presented optimization algorithm is supposed to be extended to more sophisticated control systems with multiple inputs and outputs, lumped inertial and elastic parameters, oscillating distributed networks, as well as to the modeling of the dynamic interaction of 3D elastic bodies. New mesh refinement and mesh adaptation techniques can be implemented to increase the resulting solution accuracy. Other dynamic models, for instance, viscoelastic structures are interesting for an investigation based on the proposed variational formulation. 


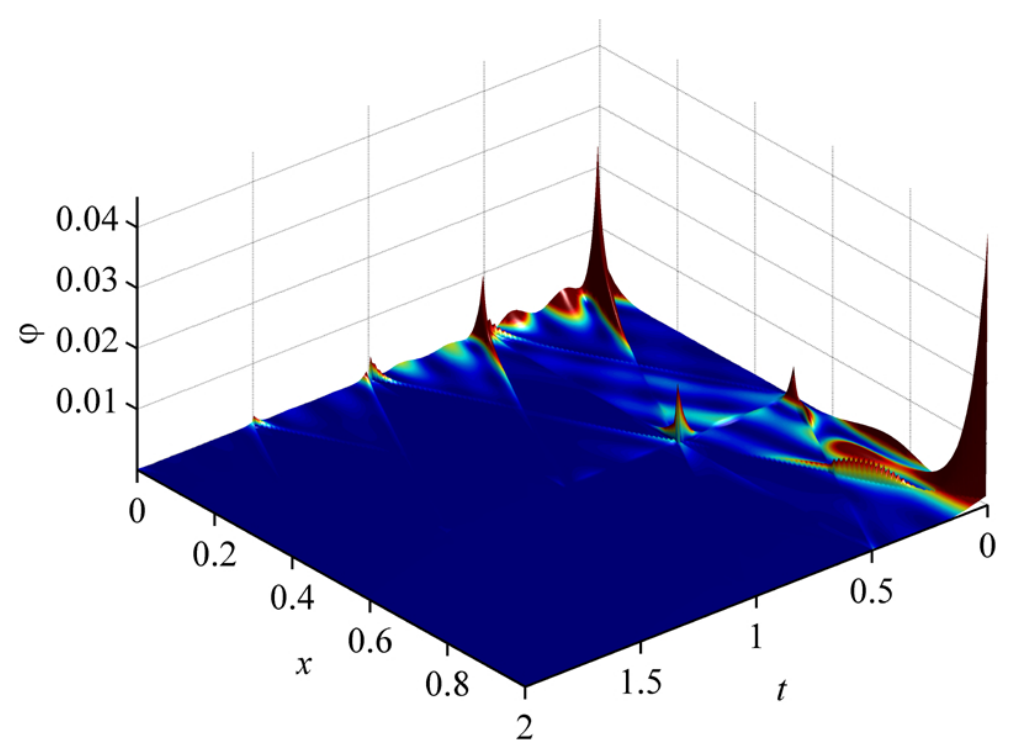

Figure 7: Error distribution $\varphi(t, x)$ for the rod motion optimized at $\gamma_{0}=0$.

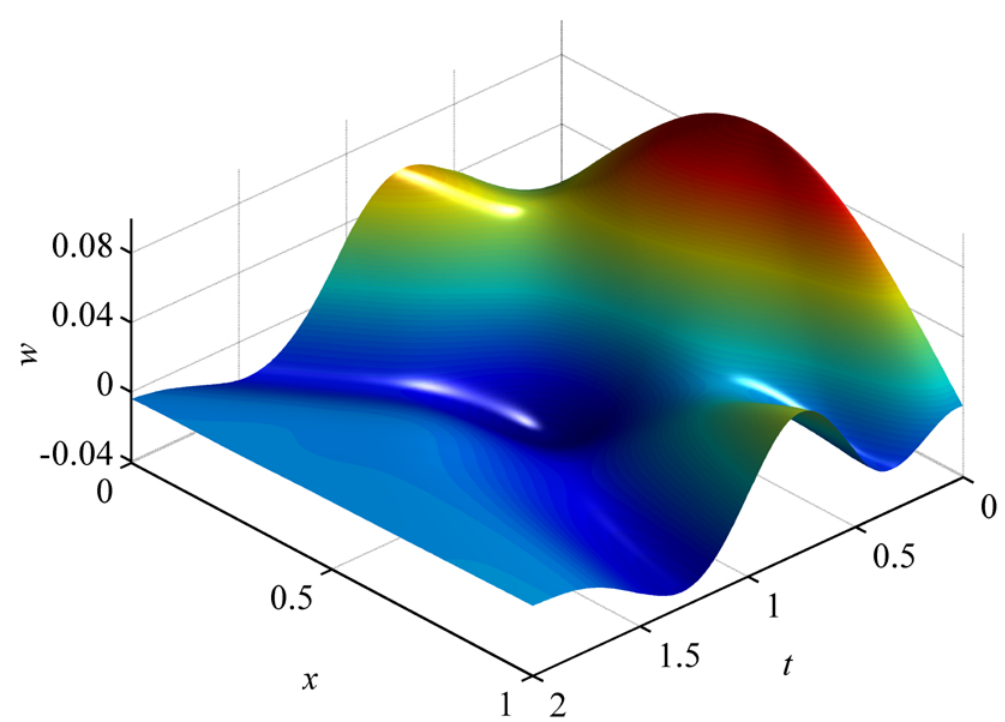

Figure 8: Optimized displacements $\tilde{w}^{*}(t, x)$ at $\gamma_{0}=10^{4}$. 


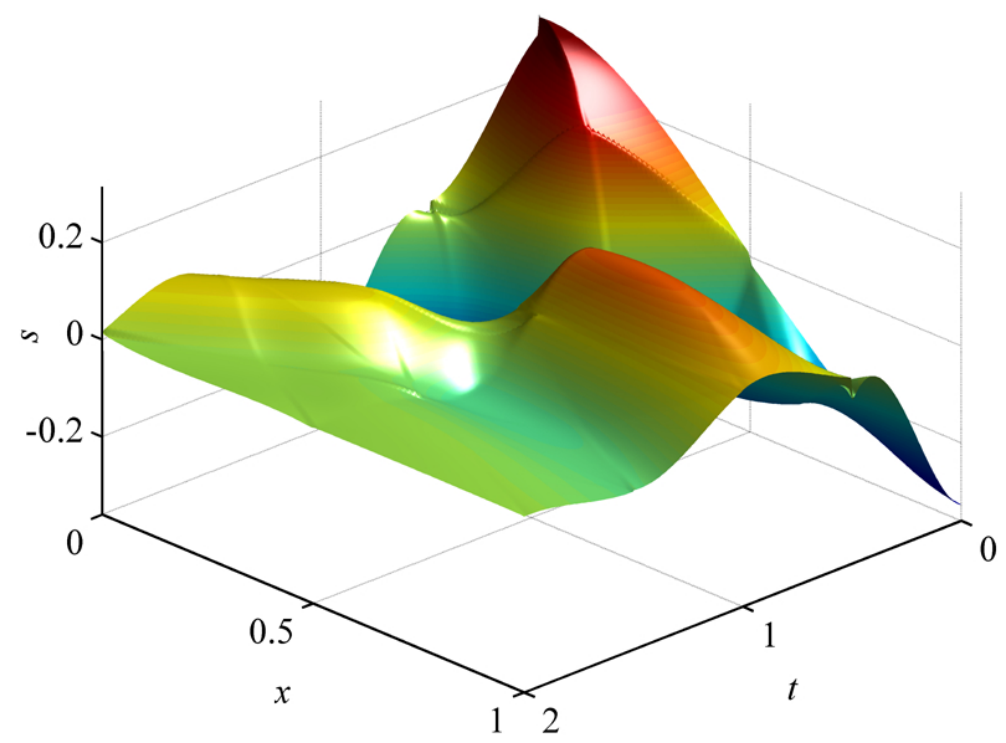

Figure 9: Optimized forces $\tilde{s}^{*}(t, x)$ at $\gamma_{0}=10^{4}$.

\section{References}

[1] Ahmed, N. U. and Teo, K. L. Optimal Control of Distributed Parameter Systems. North Holland, New York, 1981.

[2] Atluri, S. N. and Zhu, T. A new meshless local petrov-galerkin (mlpg) approach in computational mechanics. Computational Mechanics, 22:117-127, 1998. DOI: $10.1007 / \mathrm{s} 004660050346$.

[3] Balas, M. J. Finite-dimensional control of distributed parameter systems by galerkin approximation of infinite dimensional controllers. Journal of Mathematical Analysis and Applications, 114:17-36, $1986 . \quad$ DOI: 10.1016/0022-247x(86) 90062-4.

[4] Banks, S. P. State-Space and Frequency-Domain Methods in the Control of Distributed Parameter Systems. London: Peregrinus, London, 1983.

[5] Belytschko, T., Lu, Y. Y., and Gu, L. Element-Free Galerkin Method. International Journal for Numerical Methods in Engineering, 37:229-256, 1994.

[6] Butkovsky, A. G. Optimal Control of Distributed Parameter Systems. Elsevier, New-York, 1969. 
[7] Chernousko, F.L. Control of elastic systems by bounded distributed forces. Applied Mathematics and Computation, 78:103-110, 1996. DOI: 10.1016/0096-3003(96)00001-x.

[8] Christofides, P. D. Nonlinear and Robust Control of PDE Systems: Methods and Applications to Transport-Reaction Processes. Birkhäuser, 2001. DOI: 10.1007/978-1-4612-0185-4.

[9] Curtain, R and (eds.), H. Zwart. An Introduction to Infinite-Dimensional Linear Systems Theory. Springer Verlag, New York, 1995.

[10] Donnell, L. H. Beams, Plates and Shells. McGraw-Hill, New York, 1976.

[11] Gerdts, M., Greif, G., and Pesch, H. J. Numerical optimal control of the wave equation: optimal boundary control of a string to rest in finite time. Mathematics and Computers in Simulation, 79(4):1020-1032, 2008. DOI: 10.1016/j.matcom.2008.02.014.

[12] Gugat, M. Optimal control of networked hyperbolic systems: evaluation of derivatives. Advanced Modelling and Optimization, 7:9-37, 2005.

[13] Gunzburger, M. D. and Bochev, P. B. Least-Squares Finite Element Methods, volume 166 of Applied Mathematical Sciences. Springer, New-York, 2009. DOI: $10.1007 / \mathrm{b} 13382$.

[14] Kostin, G. V. Modelling and optimization of controlled longitudinal motions for an elastic rod based on the ritz method. In 201814 th International Conference "Stability and Oscillations of Nonlinear Control Systems" (Pyatnitskiy's Conference) (STAB). IEEE, 2018. DOI: 10.1109/STAB.2018.8408369.

[15] Kostin, G. V. and Saurin, V. V. A variational approach to modelling and optimization in elastic structure dynamics. In Neittaanmäki, P., Repin, S., and Tuovinen, T., editors, Computational Methods in Applied Sciences, pages 259-279. Springer, 2016. DOI: 10.1007/978-3-319-23564-6_15.

[16] Kostin, G. V. and Saurin, V. V. Dynamics of Solid Structures. Methods Using Integrodifferential Relations. De Gruyter, Berlin, 2018. DOI: 10.1515/9783110516449.

[17] Krabs, W. Optimal Control of Undamped Linear Vibrations. Heldermann, Lemgo, 1995.

[18] Lagnese, J. E., Leugering, G., and Schmidt, E. J. P. G. Modeling, Analysis and Control of Dynamic Elastic Multi-Link Structures. Birkhäuser, Boston, 1984. DOI: $10.1007 / 978-1-4612-0273-8$.

[19] Leugering, G. A domain decomposition of optimal control problems for dynamic networks of elastic strings. Computational Optimization and Applications, 16:5-29, 2000. 
[20] Lewis, R. W., Nithiarasu, P., and Seetharamu, K. N. Fundamentals of the Finite Element Method for Heat and Fluid Flow. Wiley, Chichester, 2004. DOI: $10.1002 / 0470014164$.

[21] Lions, J. L. Optimal Control of Systems Governed by Partial Differential Equations. Springer-Verlag, New York, 1971. DOI: 10.1007/978-3-642-65024-6.

[22] M. Giaquinta, S. Hildebrandt. Calculus of Variations I. Springer-Verlag, Berlin, 2004. DOI: $10.1007 / 978-3-662-03278-7$. 